\title{
Cuff inflation during ambulatory blood pressure monitoring and heart rate
}

\author{
Mia Skov-Madsen \\ My Svensson \\ Jeppe Hagstrup Christensen \\ Department of Nephrology, Aarhus \\ University Hospital, Aalborg, Denmark
}

Correspondence: Jeppe $\mathrm{H}$ Christensen Department of Nephrology, Aalborg Hospital, Aarhus University Hospital, 9100 Aalborg, Denmark

Tel +459932 6630

Email jhc@dadlnet.dk
Introduction: Twenty four-hour ambulatory blood pressure monitoring is a clinically validated procedure in evaluation of blood pressure (BP). We hypothesised that the discomfort during cuff inflation would increase the heart rate (HR) measured with 24-h ambulatory BP monitoring compared to a following HR measurement with a 24-h Holter monitor.

Methods: The study population $(n=56)$ were recruited from the outpatient's clinic at the Department of Nephrology, Aalborg Hospital, Aarhus University Hospital at Aalborg, Denmark. All the patients had chronic kidney disease (CKD). We compared HR measured with a 24-h Holter monitor with a following HR measured by a $24-\mathrm{h}$ ambulatory BP monitoring.

Results: We found a highly significant correlation between the HR measured with the Holter monitor and HR measured with 24-h ambulatory blood pressure monitoring $(r=0.77, p<0.001)$. Using the Bland-Altman plot, the mean difference in HR was only 0.5 beat/min during 24 hours with acceptable limits of agreement for both high and low HR levels. Dividing the patients into groups according to betablocker treatment, body mass index, age, sex, angiotensin-converting enzyme inhibitor treatment, statins treatment, diuretic treatment, or calcium channel blocker treatment revealed similar results as described above.

Conclusion: The results indicate that the discomfort induced by cuff inflation during 24-h ambulatory BP monitoring does not increase HR. Thus, 24-h ambulatory BP monitoring may be a reliable measurement of the BP among people with CKD.

Keywords: ambulatory blood pressure monitoring, Holter monitoring, heart rate, chronic kidney disease, hypertension

\section{Introduction}

Blood pressure (BP) measuring in a clinical environment is known to distress the patient causing a rise in BP, which makes the measured value higher than expected (Perloff et al 1983; Verdecchia et al 1994; Mancia et al 1997; Verdecchia et al 1997; Staessen et al 1999), a phenomenon referred to as "white-coat hypertension". Thus, several studies have shown a 24-h ambulatory BP monitoring to be a more accurate predictor for cardiovascular disease than clinic BP, because 24-h ambulatory BP monitoring gives a truer value of the exact BP (Perloff et al 1983; Verdecchia et al 1994; Redon et al 1998; Staessen et al 1999; Imai 1999; Hansen et al 2006; Kazuo et al 2008).

However an elevation of BP might be induced by the discomfort the patient feels during cuff inflation. This discomfort might materialize into an increased heart rate (HR) as well as BP (DeQuattro and Feng 2002). Conducting a 24-h HR from a 24-h ambulatory BP monitoring and subsequently measuring HR in 24 hours with a Holter monitor enabled us to compare the two differently measured HRs. Thus, the aim of this study was to evaluate HR during 24-h ambulatory BP monitoring compared to HR from a 24-h Holter monitoring. 


\section{Methods}

\section{Study population}

Patients $(n=64)$ were recruited from the outpatient's clinic at the Department of Nephrology, Aalborg Hospital, Aarhus University Hospital (Table 1 shows the 56 patients who completed the study). Patients were eligible if they had chronic kidney disease (CKD) defined as plasma creatinine level between 1.70 and $4.53 \mathrm{mg} / \mathrm{dL}$ ( 150 and $400 \mu \mathrm{mol} / \mathrm{L}$ ) for more than 3 months. Patients with nephrotic syndrome, malignant disease, uncontrollable hypertension, or a previous renal transplant were not asked to participate. Also, patients who had experienced myocardial infarction or underwent percutaneous coronary intervention or coronary artery bypass surgery during the previous last 6 months were not included.

The study population has previously been described (Svensson et al 2004).

HR from 24-h ambulatory BP monitoring was compared with HR from 24-h Holter readings. The 24-h ambulatory BP measurement was performed first, followed by the Holter recording.

\section{Twenty-four-hour ambulatory BP monitoring}

Twenty-four-hour ambulatory BP monitoring was performed using an ambulatory BP device, TM 2412 (A\&D Co Ltd, Tokyo, Japan). The BP was measured four times per hour

Table I Baseline characteristics of the 56 patients

\begin{tabular}{ll}
\hline Women $(\mathrm{n})$ & $16(28 \%)$ \\
Men $(\mathrm{n})$ & $40(71 \%)$ \\
Age $(\mathrm{y})$ & $58 \pm 12$ \\
Body mass index $\left(\mathrm{kg} / \mathrm{m}^{2}\right)$ & $28 \pm 5$ \\
Smokers $(\mathrm{n})$ & $21(38 \%)$ \\
Antihypertensive medication $(\mathrm{n}):$ & \\
$\beta$-blocker & $25(45 \%)$ \\
Angiotensin-converting enzyme inhibitor & $22(39 \%)$ \\
Calcium channel blocker & $36(64 \%)$ \\
Diuretics & $40(71 \%)$ \\
$\geq 2$ antihypertensives & $45(80 \%)$ \\
Statins & $7(13 \%)$ \\
Type of renal disease (n): & $10(18 \%)$ \\
Glomerulonephritis & $4(7 \%)$ \\
Chronic pyelonephritis & $7(13 \%)$ \\
Polycystic kidney disease & $4(7 \%)$ \\
Diabetic nephropathy & $15(15 \%)$ \\
Hypertension & $16(29 \%)$ \\
Other &
\end{tabular}

Note: The data are expressed as number of patients (\%) or means \pm SD. during daytime ( 6 am-10 pm) and twice each hour during the night (10 pm-6 am). Recordings were included only if at least $75 \%$ of the readings were valid.

\section{Holter monitoring}

Holter-recordings were obtained in the study participants using a flash card 3-channel digital recorder with a sampling rate of $128 \mathrm{~Hz}$ (Diagnostic Monitoring, Santa Ana, CA, USA). The recordings were started in the morning hours ( $7 \mathrm{am}-10 \mathrm{am})$ when the patients attended the outpatient's clinic and terminated the following morning 24 hours later.

\section{Statistics}

Continuous data are reported as mean $\pm \mathrm{SD}$. P-values $<0.05$ was considered statistically significant. Comparison of groups was performed using nonpaired Student's t-test for continuous variables. The Pearson's correlation coefficient was given for the correlation between the two measured HRs. The degree of agreement between the two HR parameters was assessed using the Bland-Altman analysis (Bland and Altman 1986).

\section{Results}

Fifty-six patients completed the study. Five left the study because of discomfort during the 24-h ambulatory BP monitoring. Three was excluded from the study because of technical problems with the Holter monitor.

Table 2 shows the mean HR measured with 24-h ambulatory BP monitoring and with 24-h Holter monitoring. No significant differences were found between the HRs.

Figure 1A illustrates 24-hour HR measured during 24-h ambulatory BP monitoring as a function of 24-hour HR obtained from the Holter monitor. A highly significant correlation was found between these two indices $(\mathrm{r}=0,77, \mathrm{p}<0.001)$. Figure $1 \mathrm{~B}$ shows that the mean difference between the measured HRs was

Table 2 Mean heart rate measured with both 24-h ambulatory blood pressure monitoring (BP) and with the Holter monitor in all the 56 patients and in subgroups of the patients

\begin{tabular}{lll}
\hline & $\begin{array}{l}\text { Mean heart } \\
\text { rate BP }\end{array}$ & $\begin{array}{l}\text { Mean heart } \\
\text { rate Holter }\end{array}$ \\
\hline All $(\mathrm{n}=56)$ & $73 \pm 9$ & $74 \pm 10$ \\
Women $(\mathrm{n}=16)$ & $75 \pm 10$ & $74 \pm 10$ \\
Men $(\mathrm{n}=40)$ & $73 \pm 9$ & $74 \pm 10$ \\
Patients treated with: & & \\
Betablockers $(\mathrm{n}=25)$ & $68 \pm 6$ & $69 \pm 8$ \\
Calcium channel blockers $(\mathrm{n}=36)$ & $71 \pm 7$ & $72 \pm 9$ \\
\hline
\end{tabular}

Notes: No significant differences were found. Values are expressed as means \pm SD. 
A

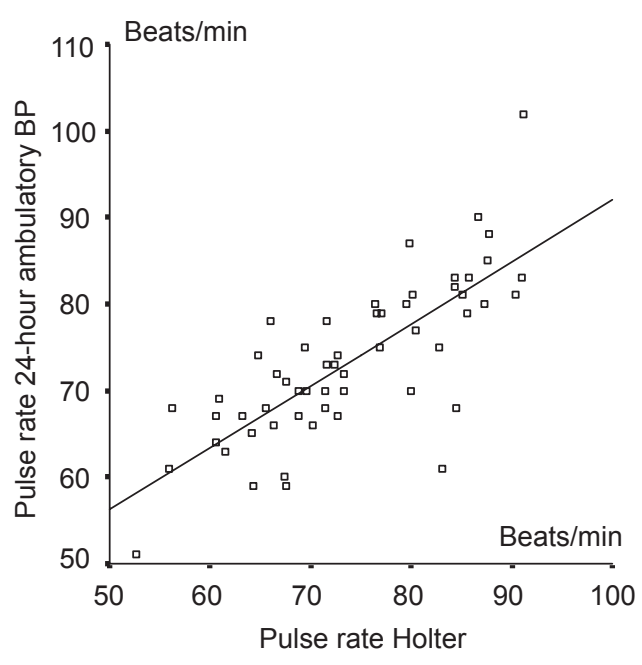

B

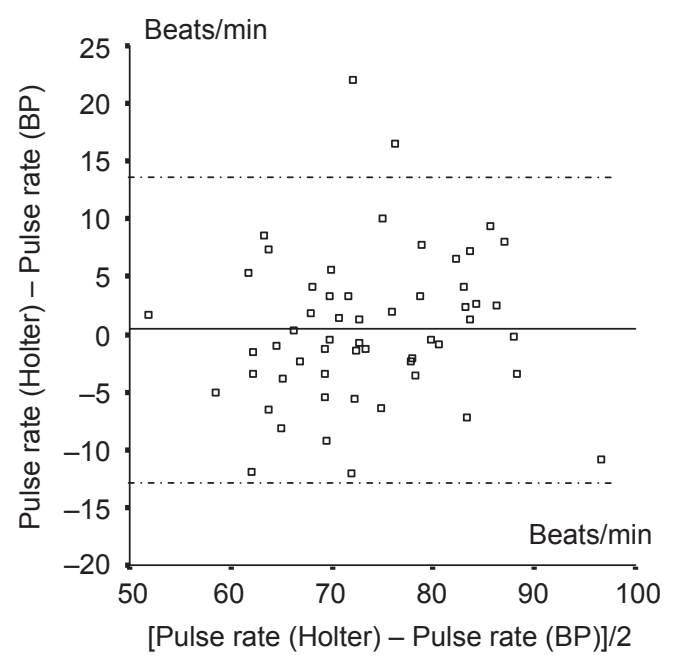

Figure I A) Shows the heart rate measured with the Holter monitor as a function of the heart rate measured with 24-hour ambulatory BP monitoring in the 56 patients $(r=0.77, P<0.00 I)$. B) Shows the Bland-Altman plot and indicates the mean heart difference (solid line) and $95 \%$ limits of agreement (dotted lines).

0.5 beats $/ \mathrm{min}$ and their corresponding $95 \%$ limits of agreement was -13.0 to 12.0 beats $/ \mathrm{min}$. Only two outliers were observed.

Figure 2A illustrates the relationship between HR measured by $24-\mathrm{h}$ ambulatory BP monitoring and the HR measured with the Holter monitor in patients treated with betablockers $(r=0.58, p<0.01)$. Figure 2B shows that the mean difference between the measured HRs was 0.5 beats $/ \mathrm{min}$ and their corresponding $95 \%$ limits of agreement was -13.5 to 12.5 beats $/ \mathrm{min}$. One outlier was seen.

Figures $3 \mathrm{~A}$ and $3 \mathrm{~B}$ show similar relationships for patients not treated with betablockers $(\mathrm{r}=0.80, \mathrm{p}<0.001)$, and a mean difference in the measured HRs of 0.4 beats/min with limits of agreement from -10.6 to 11.4 beats/min. Two outliers were found.
A

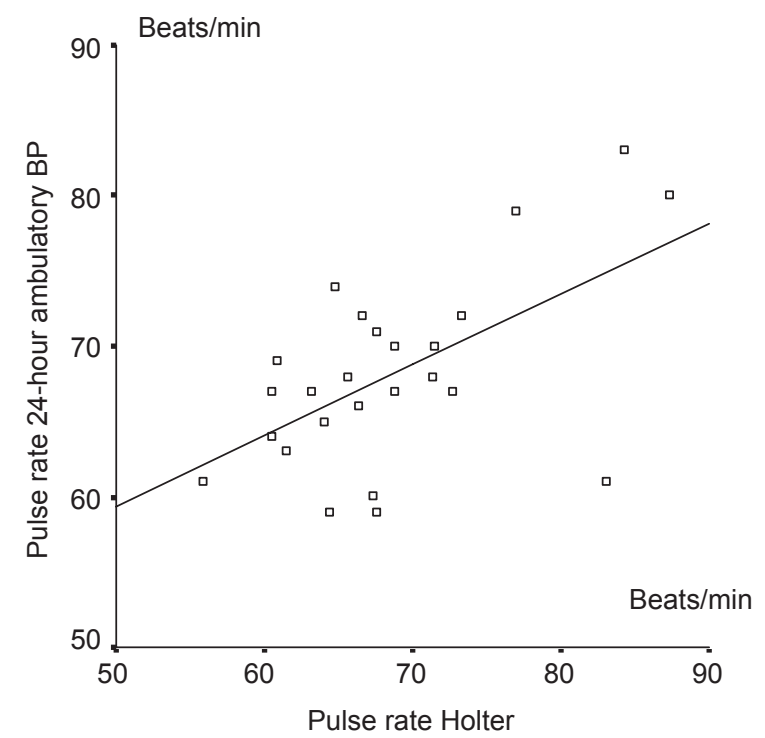

B

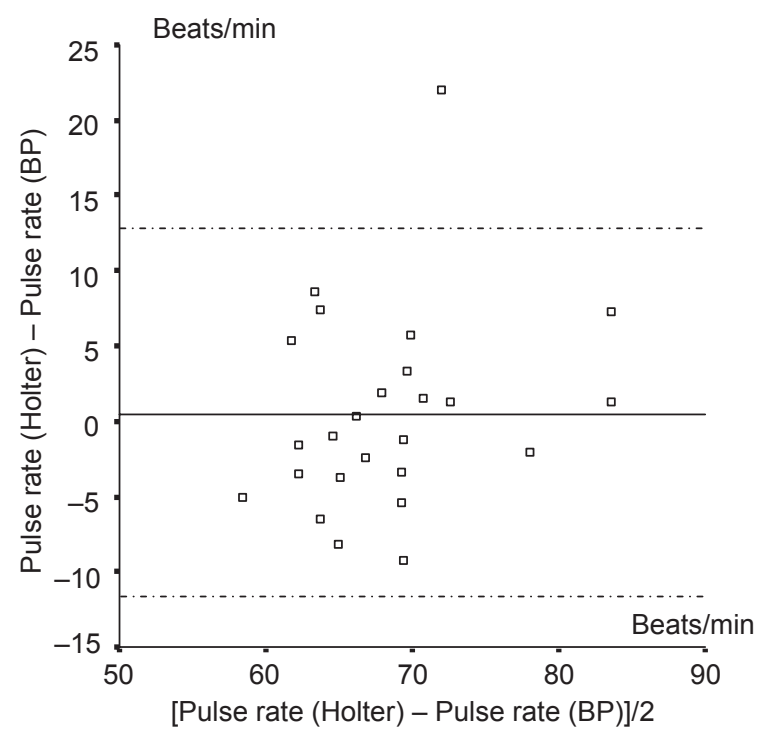

Figure 2 A) Shows the heart rate measured with the Holter monitor as a function of the heart rate measured with 24 -hour ambulatory BP monitoring in the 25 patients treated with betablockers $(r=0.58, P<0.001)$. B) Shows the Bland-Altman plot and indicates the mean heart difference $(=0.50$ beats/min $)$ with dotted lines indicating $95 \%$ limits of agreement. 
A

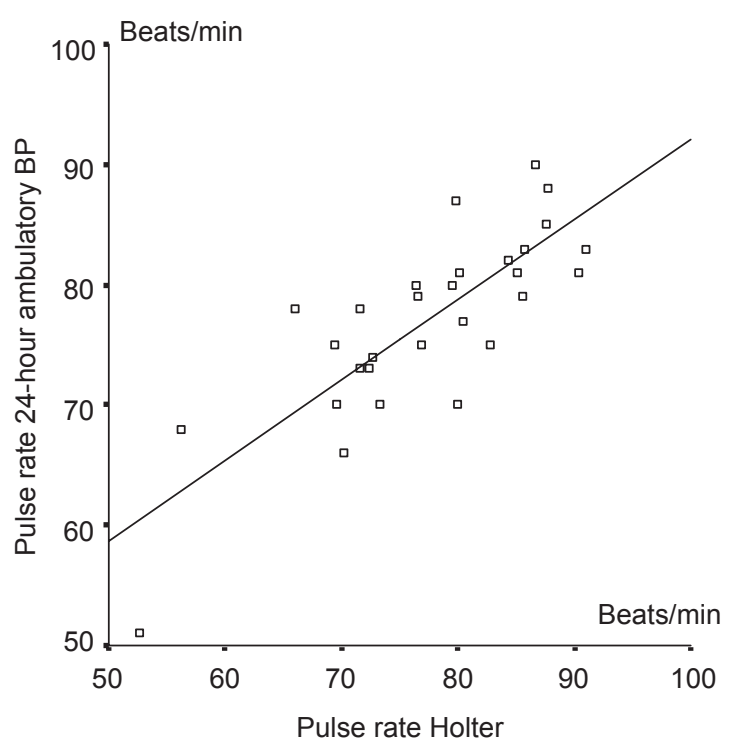

B

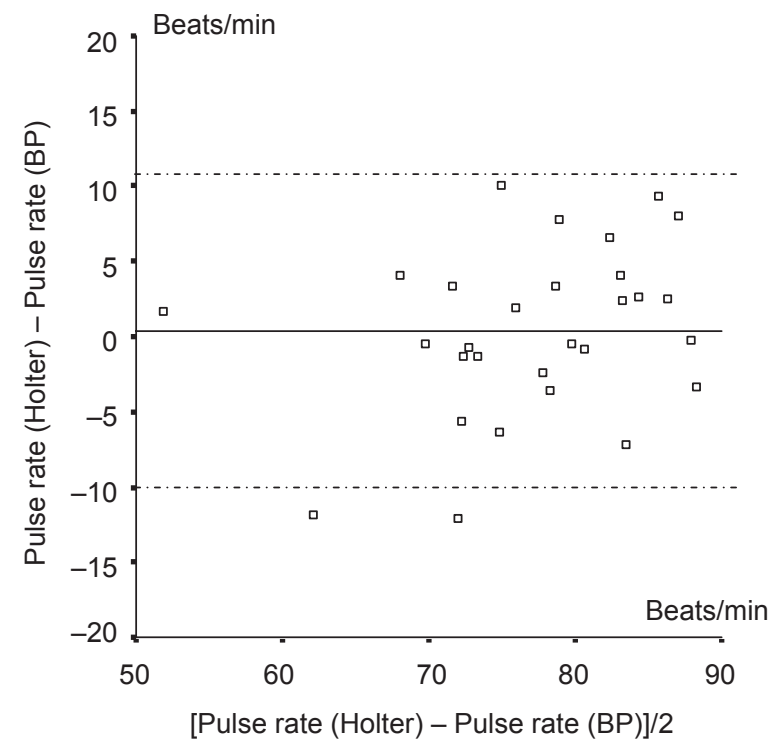

Figure 3 A) Shows the heart rate measured with the Holter monitor as a function of the heart rate measured with 24-hour ambulatory BP monitoring in the 25 patients treated with betablockers $(r=0.80, P<0.00 I)$. B) Shows the Bland-Altman plot and the solid lines indicates the mean diffrerence $(=0.40$ beats/min $)$ with dotted lines indicating $95 \%$ limits of agreement .

Further dividing the patients into groups according to body mass index, age, sex, or treated with angiotensin-converting enzyme inhibitor, statins, diuretics or calcium channel blocker also revealed significant correlations between the HRs and Bland-Altman plots comparable to the data given in the Figures (data not shown).

\section{Discussion}

This study showed a highly significant correlation between HR measured with a Holter monitor and HR measured by $24-\mathrm{h}$ ambulatory BP monitoring. Furthermore, the Bland-Altman plots revealed a low mean difference (= mean bias) of 0.5 beats/min between the two measured 24-h HRs. Furthermore, both high and low HR values were equally distributed around the mean difference line. This indicates that the possible discomfort induced when the cuff inflates is of minor importance and does not change HR. Therefore, 24-h ambulatory BP monitoring is expected to be a valid measurement of the true BP.

It is known that 24-h ambulatory BP monitoring is more closely related to target-organ damage of hypertension than the BP obtained by a physician in a clinical environment (Perloff et al 1983; Verdecchia et al 1994; Staessen et al 1999; Hansen et al 2006; Kazuo et al 2008). Even though our results indicate that 24-h ambulatory BP monitoring is very reliable, the physician should be aware of the side effects when ordering the monitoring. Some patients feel discomfort wearing the monitor and some patients experience side effects such as pain, skin irritation, noise, inconvenience in relation to work, hematoma and sleep disturbances (Beltman et al 1996; Ernst and Bergus 2003). The indications for 24-h ambulatory BP monitoring could be suspected white-coat hypertension, treatment resistance, evaluation of BP control, and borderline hypertension (Ernst and Bergus 2003). The importance of educating the patients about the benefits of undergoing 24-h ambulatory BP monitoring has been demonstrated (Ernst and Bergus 2003). The discomfort during the session may negatively influence their acceptance of undergoing the testing.

In the present study, the majority of patients were treated with antihypertensive drugs among these betablockers. Treatment with betablockers decreases HR and in addition inhibits the secretion of renin. In response cardiac output and the vascular resistance is lowered leading to a drop in BP. By inhibiting the sympathetic tone with betablockers, the triggered alerting reaction when the cuff inflates is also inhibited. Considering this, it might be assumed that the group of patients treated with betablockers would have HRs measured by 24-h ambulatory BP monitoring, which deviates less from HRs measured by the Holter, compared to the group not treated with betablockers. However, we found a stronger correlation between the two HR values in patients without betablocking therapy compared to patients treated with betablockers but the Bland-Altman plots were 
quite similar. Thirty-six of the patients were treated with group II calcium channel blockers, which predominantly have an effect on arteries and veins leading to vasodilation. Unlike other calcium channel blockers these drugs do not affect the atrioventricular node and therefore, the calcium channel blockers used in this study were not expected to affect the measured HR-values.

\section{Limitations}

Twenty-four-hour ambulatory BP monitoring and Holter monitoring were performed on two successive weekdays. The best comparable measure of the HR would be obtained if the Holter monitoring and 24-h ambulatory BP monitoring were done simultaneously. However, the 24-hours mean value of the HR obtained by the Holter monitor would then contain HR-values measured while the cuff was inflated.

In this study all the participants had CKD and it might be questioned whether the results are applicable to patients with essential arterial hypertension not receiving any medication yet.

Unfortunately, night and daytime were not discriminated by the Holter monitor and therefore we could not perform separate night and daytime analyses.

\section{Conclusion}

Our results showed that the HRs measured with 24-h Holter monitoring and 24-h ambulatory BP monitoring were quite similar. This indicates that the discomfort by the cuff inflation does not increase HR in patients with CKD.

\section{Acknowledgments}

The Danish Kidney Foundation supported this study.

\section{References}

Beltman FW, Heesen WF, Smit AJ, et al. 1996. Acceptance and side effects of ambulatory blood pressure monitoring: evaluation of a new technology. J Hum Hypertens, 10(Suppl 3):S39-S42.

Bland JM, Altman DG. 1986. Statistical methods for assessing agreement between two methods of clinical measurement. Lancet, 1:307-10.

DeQuattro V, Feng M. 2002. The sympathetic nervous system: the muse of primary hypertension. J Hum Hypertens, 16(Suppl 1):S64-S69.

Ernst ME, Bergus GR. 2003. Favorable patient acceptance of ambulatory blood pressure monitoring in a primary care setting in the united states: a cross-sectional survey. BMC Fam Pract, 4:15.

Hansen TW, Jeppesen J, Rasmussen S, et al. 2006. Ambulatory blood pressure monitoring and risk of cardiovascular disease: a population based study. Am J Hypertens, 19:243-50.

Imai Y. 1999. Prognostic significance of ambulatory blood pressure. Blood Press Monit, 4:249-56.

Eguchi K, Pickering TG, Hoshide S, et al. 2008. Ambulatory blood pressure is a better marker than clinic blood pressure in predicting cardiovascular events in patients with/without type 2 diabetes. Am J Hypertens, 21:443-50.

Mancia G, Zanchetti A, Agebiti-Rosei E, et al. 1997. Ambulatory blood pressure is superior to clinic blood pressure in predicting treatment-induces regression of left ventricular hypertrophy. Circulation, 95:1464-70.

Perloff D, Sokolow M, Cowan R. 1983. The prognostic value of ambulatory blood pressures. JAMA, 249:2792-8.

Redon J, Campos C, Narciso ML, et al. 1998. Prognostic value of ambulatory blood pressure monitoring in refrectary hypertension. Hypertension, 31:712-8.

Staessen JA, Thijs L, Fagard R, et al. 1999. Predicting cardiovascular risk using conventional vs ambulatory blood pressure in older patients with systolic hypertension. JAMA, 282:539-46.

Svensson My, Christensen JH, Sølling J, et al. 2004. The effect of n-3 fatty acids on plasma lipids and lipoproteins and blood pressure in patients with CRF. Am J Kidney Dis, 44:77-83.

Verdecchia P, Porcellati C, Schillaci G, et al. 1994. Ambulatory blood pressure, An independent predictor of prognosis in essential hypertension. Hypertension, 24:793-801.

Verdecchia P, Schillaci G, Borgioni C, et al. 1997. Prognostic significance of the white coat effect. Hypertension, 29:1218-24. 
